\title{
Comment on "Association Between DCC Polymorphisms and Susceptibility to Autism Spectrum Disorder"
}

\section{Amirhosein Meisami ${ }^{1}$}

Published online: 28 April 2020

○) Springer Science+Business Media, LLC, part of Springer Nature 2020

\section{To the Editor,}

The recent study entitled "Association Between DCC Polymorphisms and Susceptibility to Autism Spectrum Disorder" (Li et al., 2020) is published (March 2020) in the journal. The article has explored the effect of seven polymorphisms on the risk of Autism spectrum disorder.

In the abstract, the authors stated that they investigated the association between Autism spectrum disorder (ASD) susceptibility and seven SNPs in deleted in colorectal carcinoma (DCC) on the basis of a case-control study (231 ASD cases and 242 controls). Nevertheless, in Table 3 in the subjected article, the total of the genotype distributions indicated the fewer sample sizes for case and control groups in six variants. In cases when some samples are not sequenced due to technical errors, the exact number of the excluded samples should be mentioned [In Clinical characteristics section of Thakoordeen-Reddy et al. (2020) study]. In the systematic reviews, it should be reported the accurate data for reviewing and selecting the upcoming research topics by researchers. The noted errors can make a deviation in informing the sample size in future systematic reviews. Reporting the larger genotyped groups may be a conventional method in genetic studies and lead to make contradictory data.

Despite the error as mentioned above, this research had valuable results in assessing the role of rs934345, rs 17753970, rs2229082, rs2270954, rs2292043, rs2292044, and rs 16956878 polymorphisms on ASD susceptibility.

\section{References}

Li, Y., Qiu, S., Zhong, W., Li, Y., Liu, Y., Cheng, Y., \& Liu, Y. (2020). Association between DCC polymorphisms and susceptibility to autism spectrum disorder. Journal of Autism and Developmental Disorders. https://doi.org/10.1007/s10803-020-04417-3

Thakoordeen-Reddy, S., Winkler, C, Moodley, J., David, V., BinnsRoemer, E., Ramsuran, V., \& Naicker, T. (2020). Maternal variants within the apolipoprotein L1 gene are associated with preeclampsia in a South African cohort of African ancestry. European Journal of Obstetrics, Gynecology, and Reproductive Biology, 246, 129-133. https://doi.org/10.1016/j.ejogrb.2020.01.034

Publisher's Note Springer Nature remains neutral with regard to jurisdictional claims in published maps and institutional affiliations.

Amirhosein Meisami

meisami.amirhosein@yahoo.com

1 Emergency Medicine, Kermanshah University of Medical Sciences, Kermanshah, Iran 